\title{
Development of a Discriminating Dissolution Test for an Immediate Release Tablet
}

John Hempenstall, Ph.D.

GlaxoWellcome Development Ware, Hertfordsbire, United Kingdom

\section{Intraduction}

It is clearly desirable to develop laboratory tests that provide an indication of the performance of tablets in man, the so-called in vitro-in vivo correlation (IVIVC) or relationship (IVIVR) (1-4). Many attempts have been made to establish these relationships for a variety of drugs and formulations (5) with varying success. This article presents the development of a discriminating dissolution test that provides an IVIVC for an immediate release tablet undergoing clinical evaluation and describes bow this test has been used to define the 'operating window' for critical processes in the manufacture of the tablets.

\section{Solubility of the Drug Substance}

The drug substance has poor aqueous solubility at low and moderate $\mathrm{pH}$ and needs to be modified to make it more soluble (figure 1).

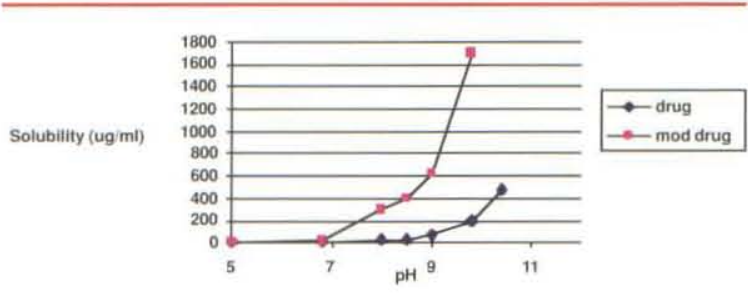

Figure 1. pH Solubility Profile for Drug and Modified Drug

This degree of solubility classifies the drug as having 'Low Solubility' as defined in the SUPAC guidelines recently produced by the FDA.

\section{Development of a Diseriminating Dissolution Test}

For tablets containing $200 \mathrm{mg}$ drug substance, a dissolution test was developed based on the USP Apparatus 2 paddle method with a weakly buffered

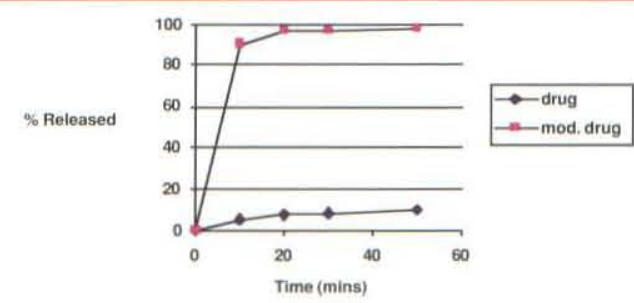

Figure 2. Discrimination Between Tablets Containing Drug and Modified Drug in the USP Apparatus 2 pH 9.0 Dissolution Test medium at $\mathrm{pH}$ 9.0. This test discriminated very well between tablets containing modified and unmodified drug substance, as shown in figure 2.

A release specification of $>80 \%$ released after 20 minutes was set for clinical batches.

However, during early clinical evaluation of the compound, different batches of tablets gave different bioavailability (table 1) despite passing the dissolution test specification (figure 3).

\section{Table 1. Relative Bioavailability of Early Clinical} Battehes

\begin{tabular}{|lcc|}
\hline \multicolumn{2}{|c|}{ Tablet Batch Number } & Relative Bioavailability \\
001 & (Standard Batch of Tablets) & 98 \\
002 & & 87 \\
003 & & 73 \\
004 & 58 \\
\hline
\end{tabular}

Clearly there was a need to develop a more discriminating dissolution test method. Simply reducing the $\mathrm{pH}$ in the USP Apparatus 2 paddle method did not work due to insufficient solubility
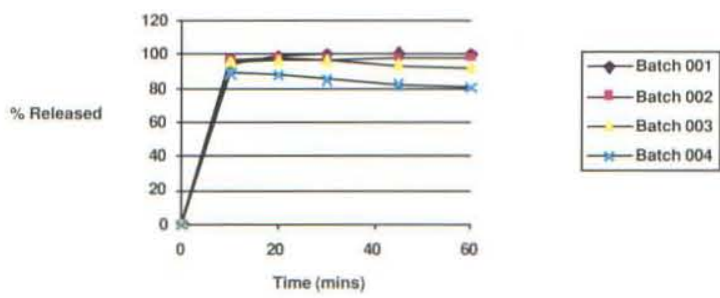

Figure 3. Dissolution Profiles of Early Clinical Batches in USP Apparatus $2 /$ pH 9 Test 


\section{Diseriminating Dissolution Test....ent.}

of the modified drug substance (figure 1). Instead, an alternative test method was developed using the USP 4 flow-through apparatus, which differs from the USP Apparatus 2 in having a much smaller dissolution cell (approx. $16 \mathrm{~mL}$ compared to $900 \mathrm{~mL}$ ) and fresh dissolution medium constantly contacting the dosage form thus maintaining 'sink' conditions throughout the test.

The batch of standard tablets (batch 001) was used to assess the effects of several test variables on the dissolution rate, the most important one being $\mathrm{pH}$ (figure 4).

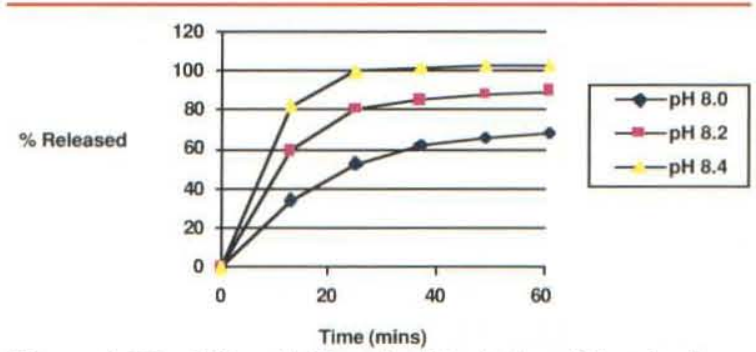

Figure 4. The Effect of $\mathrm{pH}$ on the Dissolution of Standard Tablet Batch 001 in the USP 4 Flow-through Dissolution Test

$\mathrm{pH} 8.2$ was chosen to provide high release for standard tablets after 30 minutes and a discriminating test that would indicate tablet batches that released drug substance faster as well as slower than standard.

This USP Apparatus 4 test provided excellent discrimination between tablets containing modified and unmodified drug substance (figure 5) and also discriminated well between the clinical batches that had low bioavailability (figure 6).

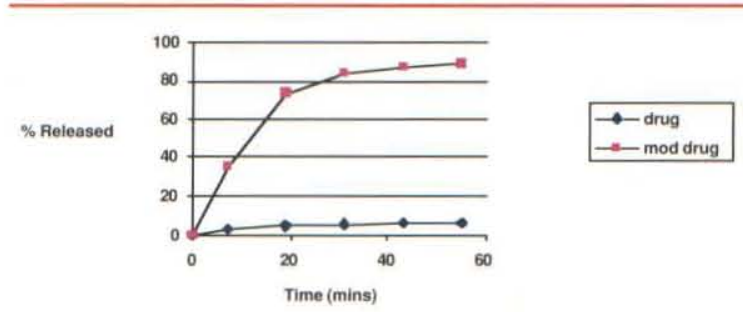

Figure 5. Discrimination Between Tablets Containing Drug and Modified Drug in the USP Apparatus 4 Dissolution Test

These data can be transformed into a graph showing the correlation between relative bioavailability and dissolution rate (figure 7).
Although not linear, there is a clear relationship between \% released after 30 minutes and bioavailability, all four batches showing the correct rank order. From figure 7, a specification limit of $>55 \%$ released after 30 minutes was set for acceptable release of drug from the tablets. This corresponded to tablets that gave $90 \%$ relative bioavailability in man.

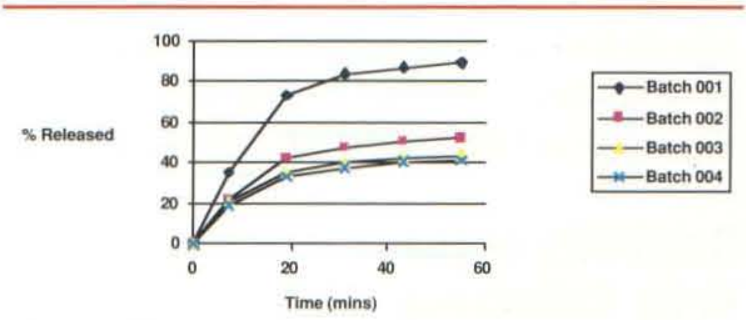

Figure 6. Dissolution Profiles of Early Clinical Batches in USP Apparatus 4 Test

The relationship shown in figure 7 was confirmed by more recent clinical batches shown to have a relative bioavailability of 94 to $101 \%$ in man and a release of 70 to $80 \%$ after 30 minutes in the USP Apparatus 4 test.

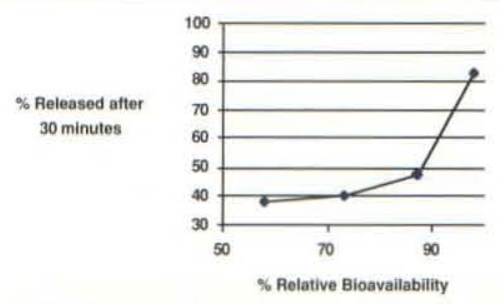

Figure 7. Correlation Between \% Released After 30 minutes in the USP Apparatus 4 Dissolution Test and Relative Bioavailability in Man

\section{Use of Discriminating Test to Define Process 'Dperating Window'}

Having developed a discriminating dissolution test that demonstrated a good in vitro-in vivo relationship, it was used to look at critical parameters for the tablet manufacturing process, such as mill speed and compression force. Figure 8 shows the effect of mill speed on dissolution rate, indicating that a mill speed 
of at least 4500 is required to produce tablets with an acceptable dissolution rate of $>55 \%$ after 30 minutes.

From such studies, the 'operating window' for the entire process at the manufacturing scale is being defined.

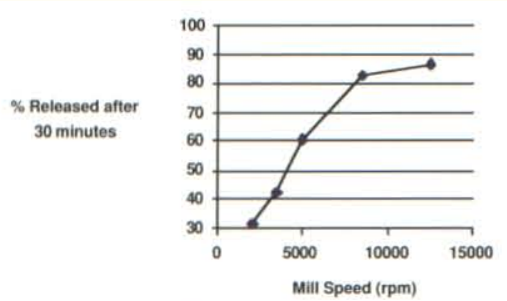

Figure 8. Effect of Mill Speed on Dissolution Rate in The USP Apparatus 4 Test

\section{Conclusion}

A dissolution test that shows an in vitro-in vivo correlation has been developed using the USP Apparatus 4 flow-through dissolution test. This discriminating test has been a powerful tool in defining the 'operating window' for the process and has obviated the need to conduct expensive and time consuming bioequivalence studies every time a process change has been conducted.

\section{References:}

1. Pharm. Forum, July-August 1988, p.4160

2. FIP Guidelines for Dissolution Testing of Solid

Oral Products, Pharm Ind., 57, 5, 362-369 (1995)

3. Dissolution Technologies 3 (1), Feb. 1996

4. Drug Information Journal, 29, 903-915, 1995

5. Drug Information Journal, 29, 893-902, 1995 